\title{
Structure of Vibriophage D10 Tail Sheath Revealed by Electron Microscopy and Computational Image Processing
}

\author{
Anindito Sen Amar N. Ghosh \\ Division of Electron Microscopy, National Institute of Cholera and Enteric Diseases, Kolkata, India
}

\section{Key Words}

Electron microscopy $\cdot$ Helical image processing •

Vibriophage D10 • Tail sheath $\cdot$ Single-particle analysis

\begin{abstract}
Vibriophage D10, a member of the Vibrio cholerae O-1El-Tor phage typing scheme, is used to detect the spread of the cholera epidemic and belongs to the Myoviridae family. The outer sheath of the tail of vibriophage is highly contractile in nature. We have used electron microscopy and computational image-processing techniques to determine the structure of this contractile tail sheath. The three-dimensional density map of the tail sheath reveals the presence of $\sim 35 \AA$ long and $\sim 25 \AA$ wide protrusions, extending out of the tail structure. The electron micrographs revealed that the tail sheaths of a considerable number of D10 phage particles undergo axial compression up to $51 \%$ at almost neutral $\mathrm{pH}$ (7.2) and at room temperature $\left(20^{\circ}\right)$. We find that the genome of the phage particles is ejected out when the tail sheath of the phage particles are deliberately made to contract by subjecting them to a surrounding environment of $\mathrm{pH}$ 10.5. We infer that the contraction of the tail sheath is responsible for
\end{abstract}

the loss of the phage genome even at neutral pH and room temperature. This may be a plausible reason for the unusual behavior of rapid decline of the phage within a span of 48$72 \mathrm{~h}$ of its preparation.

Copyright $\odot 2010$ S. Karger AG, Basel

The pathogenic bacterium Vibrio cholerae is one of the major causative agents of acute diarrheal infection, which results in loss of human life in great numbers around the globe. Extensive microbiological, biochemical and genomic research on this dreadful bacterium over the last few decades has led to the development of an advanced phage typing scheme, which helps to track down the outbreak and spread of the disease cholera. A new phagetyping scheme of $V$. cholerae O-1 biotype El Tor was developed in 1993 with the introduction of 5 new phages, namely, N4, S5, S20, M4 and D10. Preliminary characterization of these phages was carried out by Chattopadhyay et al. [1]. One of the typing phages, D10, contains a $32-\mathrm{kb}$ genome encapsulated in an icosahedral head of diameter $520 \pm 23 \AA$ and was characterized using biochemical and electron microscopic techniques $[2,3]$. The size of the vi-

\section{KARGER}

Fax +41613061234 E-Mail karger@karger.ch www.karger.com
(C) 2010 S. Karger AG, Basel

0300-5526/11/0541-0044\$38.00/0

Accessible online at:

www.karger.com/int
Amar N. Ghosh

Division of Electron Microscopy, National Institute of Cholera and Enteric Diseases P33 CIT Road Scheme XM

Beleghata, Kolkata 700010 (India)

Tel. +91 332363 3864, Fax +91 332370 5066, E-Mail ghoshan@icmr.org.in 

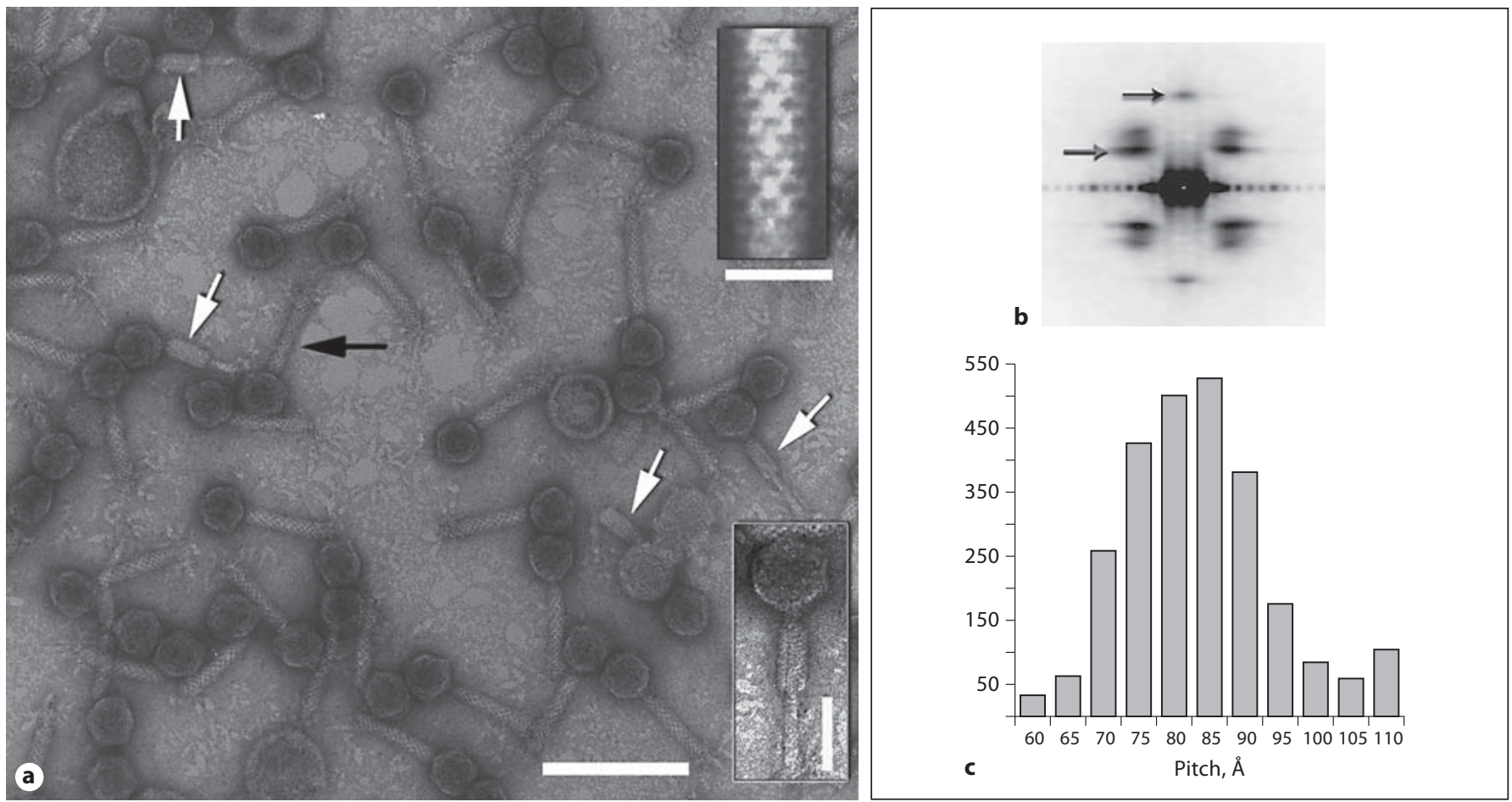

Fig. 1. Electron micrograph and computational image processing of D10 tail sheath. a Electron micrograph of D10 vibriophage stained with $1 \%$ uranyl acetate. The micrograph shows the presence of virion particles with contracted and uncontracted tail sheaths, marked by the white and black arrows respectively. Bar $1,000 \AA$. The inset at the top right hand is a global average to the uncontracted tail sheath particles. Bar $100 \AA$. The inset at the bottom right is a D10 virion particle with its outer sheath compressed, exposing the inner tube. Bar $250 \AA$ A. b Global average power spectrum of the tail sheath of D10 virion particles generated from 363 particles with varying lengths in the range of $800-1,000 \AA$. The black and grey arrows indicate the axial rise and helical pitch at $1 / 32$ and $1 / 84 \AA$, respectively. c Graphical representation of the result of classification of the tail sheath segments based on the variability of pitch. The $\mathrm{x}$ axis represents measure of the helical pitch and $y$ axis represents the number of sheath segments. rion particles reported in earlier papers was slightly different as measurements were done on magnified prints. These studies also revealed that the phage genome is a non-permuted dsDNA with cohesive ends. The GC-rich end of dsDNA in the D10 phage head disrupted under control conditions was found to be attached to its tail and it was inferred that during packaging of the DNA the GCrich end is packaged last and stays in close proximity to the phage tail [2]. In the present study electron micrographs of the D10 vibriophage reveal that the outer tail sheath of a small population of virion particles has undergone axial contraction from a normal length of $810 \pm$ 13 to $390 \pm 5 \AA$ (fig. 1a). The contracted forms were also seen when the samples were stained with phosphotungstic acid and ammonium molybdate, indicating that the compression of the outer tail sheaths was not a consequence of the use of uranyl acetate stain. The contraction causes the thickness of the outer tail sheath to increase from $180 \pm 7$ to $200 \pm 10 \AA$ and exposes the $80 \pm 7 \AA$ thick inner tube which is attached to the 5 -fold symmetry axis of the phage head (fig. 1a, inset bottom right). To the best of our knowledge tail sheaths of any other lytic vibriophage (like AS1) do not show such behavior at room temperature $\left(20^{\circ}\right)$, at near-neutral $\mathrm{pH}(7.2)$ or in the absence of any harsh chemical like urea [4; Sen and Ghosh, unpublished results]. In order to elucidate the structure of this contractile tail sheath, we have generated a 3-dimentional (3D) view of this biopolymer using different algorithms on electron microscope images of D10.

A highly purified and concentrated D10 phage stock was prepared using MAK757 as the propagating strain and was maintained as described elsewhere $[2,5]$. One of the big challenges faced with the sample preparation and maintenance was the brisk decline in the titer in the order of $10^{3}-10^{4}$ particles/ml within a short time span of 48 $72 \mathrm{~h}$ [Ghosh, unpublished observation]. About $5 \mu \mathrm{l}$ of the 


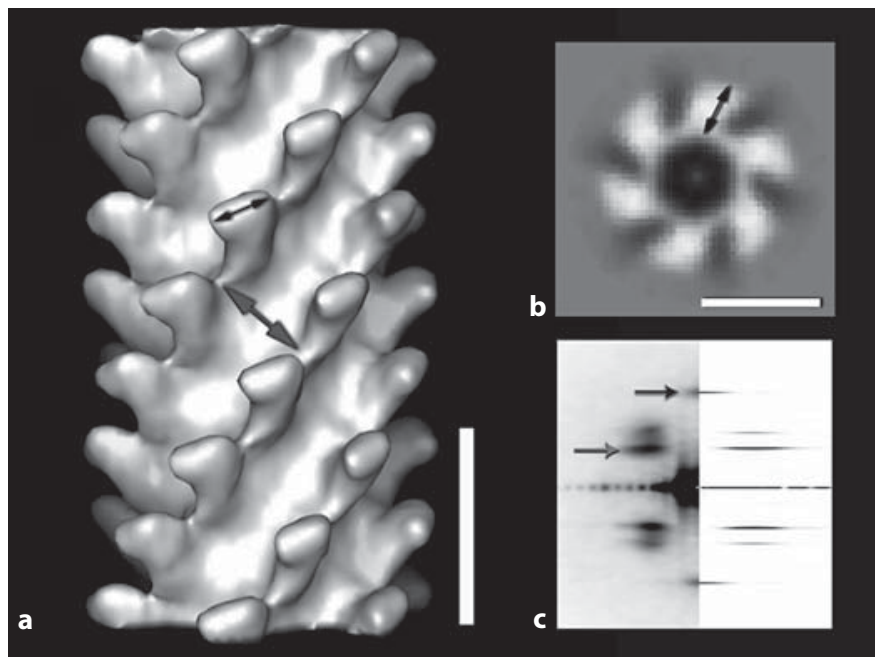

Fig. 2. 3D image reconstruction and power spectrum from the computed density map of the tail sheath of the D10 virion particles. a Iso-surface representation of the density map of the D10 tail sheath showing $\sim 25$ - $\AA$ wide protrusions (black arrow) arranged in a systematic helical manner. The distance between two layers of protrusions (grey arrow) is $\sim 40 \AA$. Bar $100 \AA$. b Grey scale image of the top view of the D10 tail sheath. A volume, with diameter equal to that of the inner core, is computationally removed for better analysis of the architecture of the D10 sheath. The protein density is white. The length of the protrusions is $\sim 35$ $\AA$. Bar 100 Å. c Comparison of the global power spectrum (on the left hand side) to that generated from the final density map (on the right hand side). The two power spectrums match each other nicely as indicated by the back and grey arrows representing the axial rise and the helical pitch of the D10 tail sheath. The result validates the accuracy of the computational methods used in the present work.

phage suspension in $0.05 \mathrm{M}$ Tris- $\mathrm{HCl}(\mathrm{pH} 7.2)$ and $0.02 \mathrm{M}$ $\mathrm{MgCl}_{2}$ (TM) buffer was placed on 300-mesh nickel grids stabilized with a thin layer of carbon and was then subjected to one of the following stains at room temperature $\left(20^{\circ}\right)$ : (i) $1 \%(\mathrm{w} / \mathrm{v}$ ) uranyl acetate ( $\mathrm{pH}$ unadjusted); (ii) $1 \%$ (w/v) phosphotungstic acid, or (iii) $1 \%(\mathrm{w} / \mathrm{v})$ ammonium molybdate. However, the best results were obtained with uranyl acetate. Images were recorded on Kodak 4489 plate films in a Philips 420T transmission electron microscope at an accelerating voltage of $120 \mathrm{kV}$ and at a magnification of $\times 60,000$. The magnification was calibrated with beef catalyze crystals (Pelco). The negatives were then scanned using a Nikon $9000 \mathrm{CoolScan}$ at 2,000 d.p.i. resulting at a pixel size of $2.1 \AA$.

A total of 363 individual tail particles (fig. 1a, black arrow) were 'boxed' out from 11 micrographs using the program 'helixboxer' of the software package EMAN [6].
The central helical section containing the outer sheath was then extracted from the individual tail particles and used to generate global power spectrum using the algorithm 'bctf' of the BSOFT package [7]. The power spectrum showed a clear meridional reflection positioned at $1 / 32 \AA$ (fig. 1b, black arrow) that represents the axial rise $(\Delta \mathrm{x})$ of a subunit forming the helical assembly. The first strongest reflection parallel to the equator representing the helical pitch (fig. 1b, grey arrow) is positioned at $1 / 84 \AA$. These two parameters were used to calculate the angular rise $(\Delta \varphi)$ of a subunit which is equal to $\sim 137.1^{\circ}$. The central helical sections, containing the outer sheath images were then computationally chopped into 2,606 overlapping segments and the whole dataset was binned by a factor of $2 \times 2$ to accelerate the image processing which resulted in a final pixel of $4.2 \AA$ and box size of 64 $\times 64$ pixels. An averaged image of these sheath segments was generated using 'classalign2' of the EMAN software package and is shown in the inset at the top right of figure 1a.

3D image reconstruction of the tail sheath particles was initiated using iterative helical real-space reconstruction algorithm using the helical parameters $(\Delta x, \Delta \varphi)$ described earlier [8]. After several rounds of iterations, the view down the helical axis of the resulting density maps clearly indicated the presence of 6-fold point-group symmetry. This symmetry was then applied in the subsequent iterations. The reconstruction failed to converge even after 35 iterations to a stable solution, which is indicative of the presence of heterogeneity in the dataset. In order to sort out this heterogeneity, we generated 11 different density maps of the tail sheath with the helical pitch ranging from 60 to $110 \AA$ at a step size of $5 \AA$. A set of 36 projections at an angular step size of $10^{\circ}$ was generated for each of these 11 density maps. The sheath segments were then classified against a total of 396 projections by employing the algorithm 'APNQ' of the SPIDER software package [9]. The histogram showing the distribution of the sheath segments in the bins with different pitch values is presented in figure $1 \mathrm{c}$. The plot reveals that the majority of the segments belong to the groups 80 and $85 \AA$ A. Segments, belonging to these 2 groups, were then computationally extracted out from the raw dataset, combined together and were used for further image processing. Since the reconstruction was calculated from a dataset of negatively stained particles, we do not expect the resolution of the density map to be anything better than $17 \AA$ at a Fourier shell correlation cutoff of 0.5 . Thus, we filtered the final density map to $17 \AA$ to reduce noise generated from signals of high frequencies. Figure $2 \mathrm{a}$ is 
an iso-surface representation of D10 tail sheath. The density map reveals the presence of $\sim 35 \AA$ long and $\sim 25 \AA$ wide protrusions sticking out of the central tail body (fig. 2a, b). The distance between the layers of protruding regions (fig. 2a, grey arrow) is $\sim 40 \AA$.

In order to investigate any possible morphological change the phage particles might undergo due to the contraction of the outer tail sheath, we treated the D10 virions with high $\mathrm{pH}$ (10.5) buffer. This resulted in the contraction of the outer tail sheath of nearly all the phage particles by $\sim 51 \%$ in length and exposed the inner tail tube. These virion particles, when subjected to platinum metal shadowing, revealed ejection of their genomes from the capsids (fig. 3a). The methodology for this experiment is described elaborately by Chakrabarti et al. [2]. The result of this experiment points towards a possible situation where the phage genome is ejected from its capsid due to the contraction of the outer tail sheath. We believe that the phage particles, whose outer sheaths are contracted even at near-neutral $\mathrm{pH}$ (7.2) and room temperature $\left(20^{\circ}\right)$, due to reasons unknown to us, undergo the same fate and loose their genomes leading to a decline in phage concentration.

To test the validity of the computational process, we generated a $2 \mathrm{D}$ projection of the final density map (fig. $3 \mathrm{~b}$ ) and compared it to the global average (fig. 1a, top right inset). We noticed a great deal of similarity with each other. We iteratively aligned and classified the tail sheath segments by multivariate statistical analysis using the 'refine2d.py' algorithm of EMAN package which generated 37 different class averages (fig. 3c) [6, 10]. These class averages were then compared to a set of 90 projections from the final reconstruction generated at an angular step of $4^{\circ}$ and a very good match was observed. A power spectrum was generated from the $2 \mathrm{D}$ projection of the final of the density map (fig. 3b) and was compared with the global reconstruction (fig. 2c). The comparison revealed a near-perfect match thus validating the accuracy of the reconstruction process.

In order to view the variation in the helical pitch of the sheath particles among the different bins shown in figure 1c, we calculated power spectrums of the tail sheath particles belonging to the groups with helical pitches of 75 and $90 \AA$, respectively, and compared them side by side. The comparison clearly shows a difference in the helical pitch, although very weak due to the very small number of particles computed, between the particles belonging to the 2 groups and thus confirms the accuracy of the computation process. Whether the highly contractile nature of the D10 tail sheath is related to the short adsorption
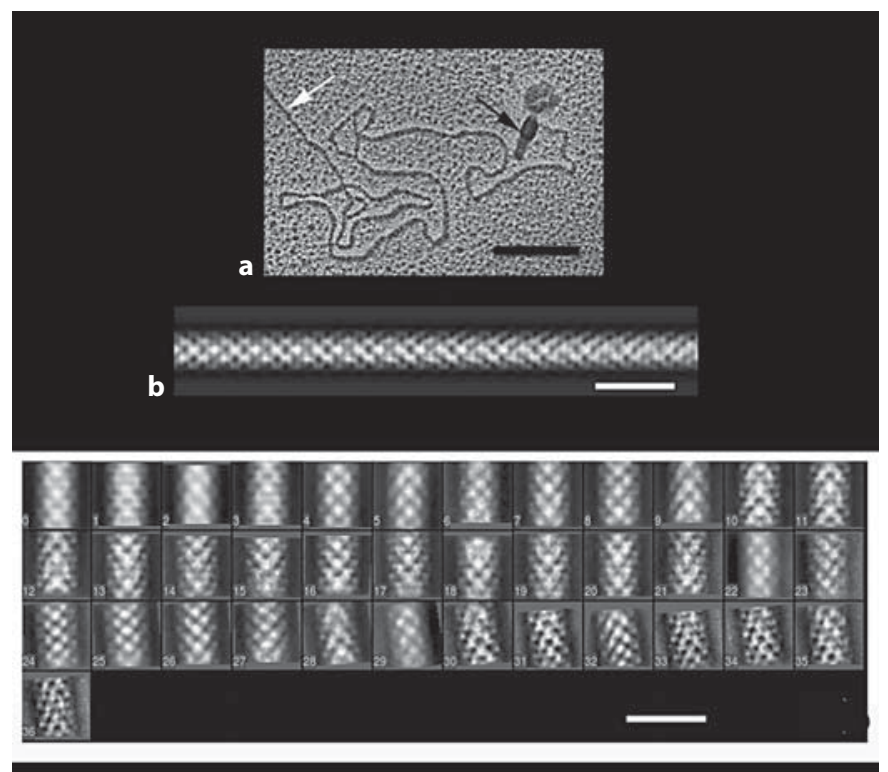

c

Fig. 3. Electron micrograph showing the effect of $\mathrm{pH}$ on D10 virion particle and class average of tail sheath segments. a Effect of $\mathrm{pH}$ on the $\mathrm{D} 10$ virion particle. The virion particles were subjected to a buffer of $\mathrm{pH} 10.5$ and then mounted on grids. These particles were then subjected to platinum shadowing and examined at the TEM. At pH 10.5 the tail sheath contracts (black arrow), exposes the inner core and releases the phage genome marked by a white arrow. Bar 1,000 A. b A 2D projection of the density map of the tail sheath generated using the program 'bproject' of the software package BSOFT [7]. The projection shows remarkable resemblance to the global average of the tail sheath shown in the inset at the top right in figure la thus indicating the accuracy of the image processing. Bar $200 \AA$ A. c A reference-free class average generated from 2,606 tail sheath segments of a size of $64 \times 64$ pixels resulting in 37 classes. The different classes showed a great deal of similarity with the projections generated from the final density map of the tail sheath validating the correctness of the reconstruction process. Bar $200 \AA$.

and injection time of the genome to the host bacterium $V$. cholerae in comparison to other lytic vibriophages leading to a high titer phage stock in a short period of time will be an interesting problem to study. The possibility of the viral genome being ejected out of the phage during the compression of the tail sheath under normal conditions cannot be ruled out and perhaps may be the reason for the rapid decline in the phage titer. What triggers the compression of the tail sheath under normal conditions (near-neutral $\mathrm{pH}$ and room temperature) is completely unknown to us. These issues can only be addressed by further biochemical and molecular biological studies of this very fascinating vibriophage. 


\section{References}

$>1$ Chattopadhyay DJ, Sarkar BL, Ansari MQ, Chakrabarti BK, Roy MK, Ghosh AN, Pal SC: New phage typing scheme for Vibrio cholerae $\mathrm{O} 1$ biotype El Tor strains. J Clin Microbiol 1993;31:1579-1585.

2 Chakrabarti BK, Chattopadhyay DJ, Ghosh AN: Vibriophage D10 contains non-permuted DNA with cohesive ends. J Gen Virol 1993;72:2749-2752.

3 Chakrabarti BK, Si K, Chattopadhyay D: Characterization of Vibrio cholerae EI Tor typing phage D10. J Gen Virol 1996;77:28812884.
4 Sen A, Ghosh AN: New Vibrio cholerae O1 biotype ElT or bacteriophages. Virol J 2005 $2: 28$

5 Adams MH: Bacteriophages. New York, Interscience, 1959.

6 Ludtke SJ, Baldwin PR, Chiu W: EMAN semiautomated software for high-resolution single-particle reconstructions. J Struct Biol 1999; 128:82-97.

7 Heymann JB, Belnap DM: Bsoft: image processing and molecular modeling for electron microscopy. J Struct Biol 2007;157:3-18.

$\checkmark 8$ Egelman EH: A robust algorithm for the reconstruction of helical filaments using single-particle methods. Ultramicroscopy 2000;85:225-234.
9 Frank J, Radermacher M, Penczek P, Zhu J, Li Y, Ladjadj M, Leith A: SPIDER and WEB: processing and visualization of images in 3D electron microscopy and related fields. J Struct Biol 1996;116:190-199.

10 Zhang R, Hu X, Khant H, Ludtke SJ, Chiu W, Schmid MF, Frieden C, Lee JM: Interprotofilament interactions between Alzheimer's Abeta1-42 peptides in amyloid fibrils revealed by cryoEM. Proc Natl Acad Sci USA 2009;24:4653-4658. 